\title{
Does Visual Access When Lifting Unstable Objects Affect the Biomechanical Loads Experienced By the Spine and Shoulders?
}

\author{
THESIS
}

Presented in Partial Fulfillment of the Requirements for the Degree Master of Science in the Graduate School of The Ohio State University

\author{
By \\ Xueke Wang \\ Graduate Program in Industrial and Systems Engineering \\ The Ohio State University \\ 2017 \\ Master's Examination Committee: \\ Steven Lavender, Advisor \\ Carolyn Sommerich
}


Copyrighted by

Xueke Wang

2017 


\begin{abstract}
Objective: To explore the change of muscular and biomechanical responses in different load stability and visual access conditions during asymmetric lifting tasks.

Background: Most of the lifting biomechanics literature has focused on the lifting of stable loads. However, the handling of unstable loads was hypothesized to result in lifting behaviors that would increase the biomechanical loading of the spine and the upper extremities. It was further hypothesized that being able to view the contents of the container being lifted would mitigate the increased biomechanical response when unstable loads were lifted.
\end{abstract}

Methods: Fourteen volunteers, 8 male and 6 female lifted a wooden box containing either a stable load, bags of water, or a bowling ball that could shift during the lifting task. The box, which in half the conditions was covered and in half the conditions uncovered, was lifted from the floor from a location directly in front of the subject and then placed at approximately waist height on a surface that was on the participant's left side (90 degrees of asymmetry). Spine kinematic and kinetic measures and bilateral surface electromyographic (EMG) signals from the erector spinae, the external oblique, anterior deltoid, and biceps muscles were obtained.

Results: In some paired comparisons, it was found that when lifting stable loads the activities in the left external oblique, right biceps and anterior deltoid increased. However, the duration of the lifting tasks was found to be longer when lifting the 
unstable loads. The uncovered box decreased the activation levels of the left biceps, both anterior deltoid, and both erector spinae muscles. The frequency of load shifting was reduced when the box containing the bowling ball was uncovered.

Conclusion: Contrary to the initial hypothesis, lifting unstable loads did not increase the activities in target muscles and biomechanical loads but did result in lifts of longer duration. When lifting a very unstable load (the bowling ball in this study), having an uncovered container reduced activity in relevant muscles and reduced the likelihood of the bowling ball shifting when compared to the same task with container covered.

Relevance: In industry, there are many work situations where workers need to lift or carry unstable loads that can shift during transport. These shifting loads result in higher demands on muscles and risks to workers' health. Being able to see the unstable loads might mitigate these effects which suggest the value of transparent or translucent packaging.

Keywords: industrial design, unstable load, visual information, muscle response, spinal moments. 


\section{Vita}

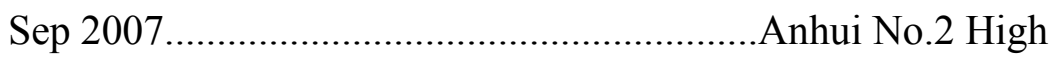

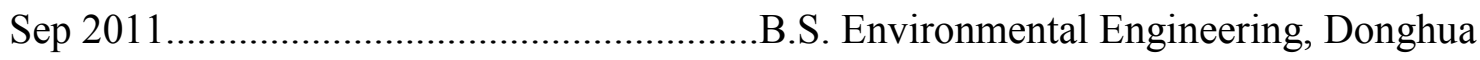

University

April 2017 ................................................... Industrial Engineering, The Ohio State

University

2015 to present ..........................................Graduate Teaching Associate, Industrial

Engineering, The Ohio State University

Fields of Study

Major Field: Industrial and Systems Engineering 


\section{Table of Contents}

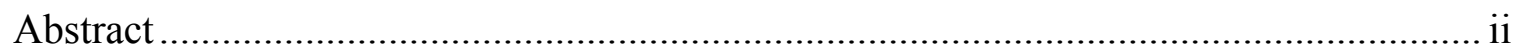

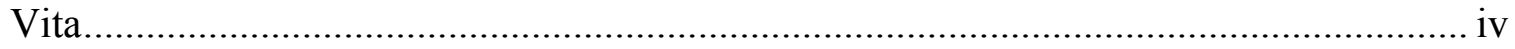

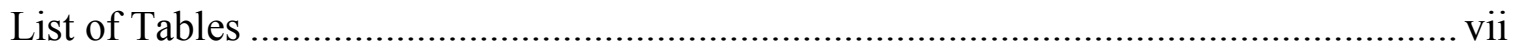

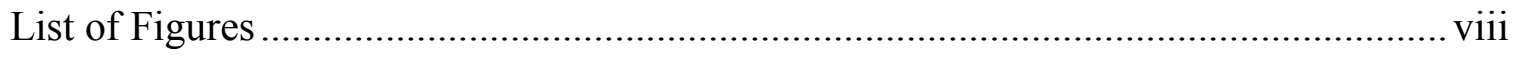

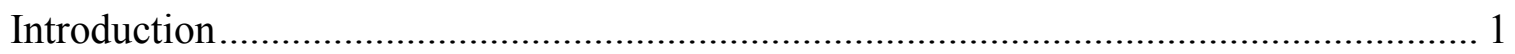

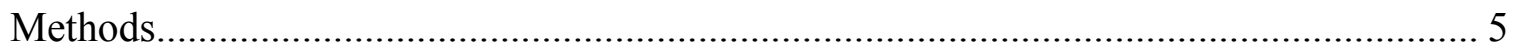

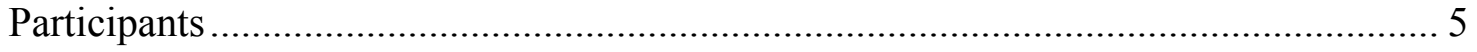

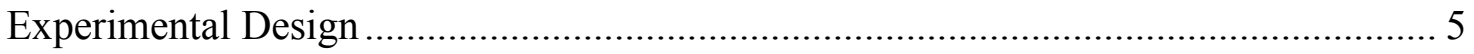

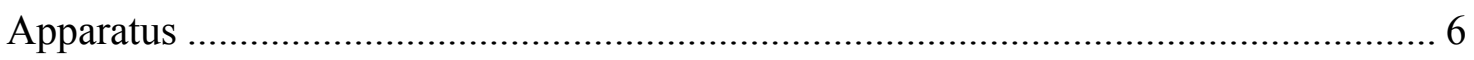

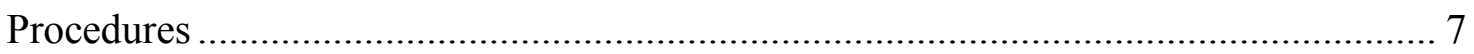

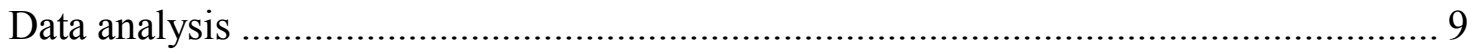

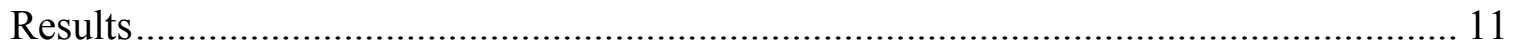

Comparison between stable and liquid load................................................... 11

Comparison between stable and potentially unstable load (Lifts where the ball did not

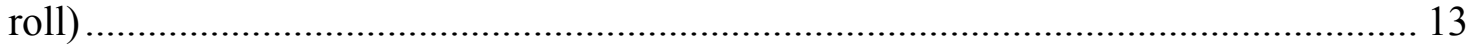


Comparison between stable and unstable load (rolling ball)

Comparison between potentially unstable (non-rolling) and unstable (rolling) load

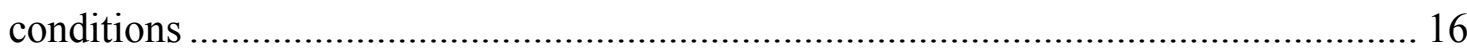

Interaction Effects ........................................................................................ 18

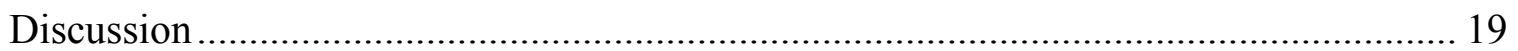

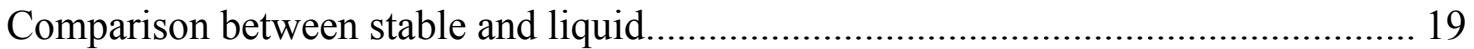

Comparison between stable and non-rolling ...................................................... 20

Comparison between stable and rolling .......................................................... 21

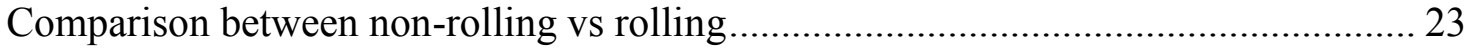

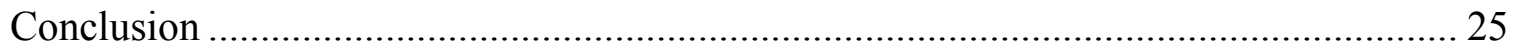

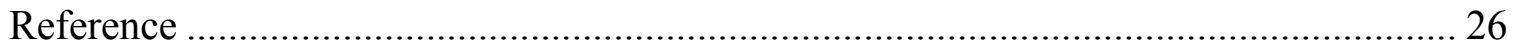




\section{List of Tables}

Table 1. Pair-wised Comparison between Different Load Stability and Visual Access

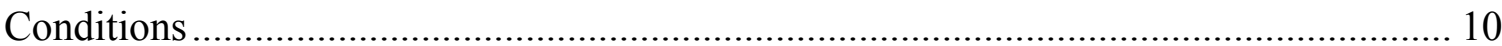




\section{List of Figures}

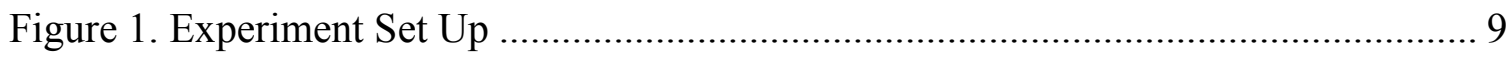

Figure 2. Visual Access Effect on Max 90 Muscle Activities for Data Collected During

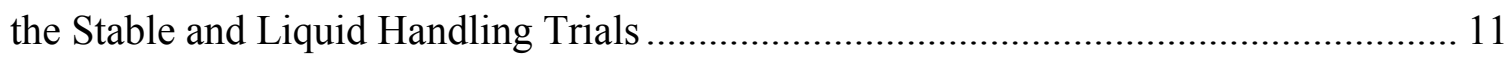

Figure 3. Visual Access Effect on Thoracic Biomechanics Responses - Stable vs Liquid

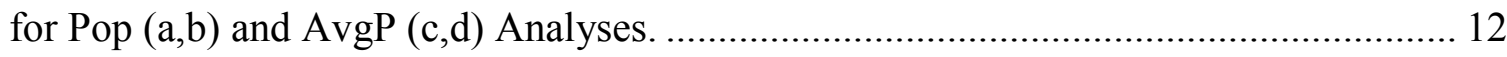

Figure 4. The Load Stability Effect on Muscle Activities - Stable vs. Non-rolling for

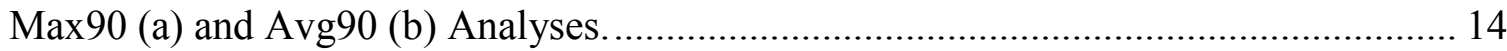

Figure 5. Visual Access Effect on Muscle Activities - Stable vs. Non-rolling for Max90 (a) and $\operatorname{Avg} 90$ (b) analyses.

Figure 6. Main Effect for Visual Access on Muscle Activities for the- Max90 (a) and Avg90 (b) Analyses (Averaged Across the Stable and Rolling Conditions) .................... 16 Figure 7. Load Stability Effect on Muscle Activity - Non-rolling vs. Rolling (Avg90) .. 17 Figure 8. Visual Access Effect on Muscle Activities - Non-rolling vs Rolling (Avg 90) 18 
Introduction

Work-related low back injury remains one of the top occupational musculoskeletal disorders in industry (BLS, 2016). According to the data from the Bureau of Labor Statistics, there were more than 155,000 back injuries in the year of 2015, among which, 16,520 cases happened in the lumbar region and caused 3-5 days away from work. Thus, work-related low back disorders are still prevalent and require more research to develop effective interventions. Based on epidemiological studies, low back injury has been associated with various manual material handling tasks, especially lifting tasks (Andersson, 1997). For lifting tasks, many studies have investigated the contribution of the magnitude of the load, the effects of reaching with the load, the repetition, the asymmetry of the lifting task, and the repetitive nature of the work (Andersson, 1997). In addition, epidemiology studies have also found that sudden loading events were associated with the onset of low back injuries (Magora, 1973; Andersson, 1981). Sudden loading events, which are loading events that occur quickly and possibly without warning, can happen in a variety of ways in the workplace. For example, nurses and physical therapists are exposed to the risk of back injury in patient handling where patients shift or start to fall and sudden maximal exertions were made in response (Owen, $1984 \&$ Molumphy, 1985). Other sudden loading scenarios could exist when handling airplane passengers' baggage, products with deficient packaging 
materials, when objects stick to one another, or when handling products where the contents can shift within the packaging. Alternatively, when the timing of a sudden loading event cannot be accurately predicted, for example with the absence of visual cue signaling the onset of the sudden loading, there could be significantly higher forces in the trunk musculature (Marras et al. 1987). Marras et al. (1987) found that when subjects had no visual or auditory cues indicating when a load would drop into a container held in the hands, normalized electromyographic signals indicative of muscle force were about 2.5 times larger than when subjects had visual cues indicating the timing of the loading event. Lavender et al. (1989), using a similar approach of a weight dropping into a box, found that the magnitude of the trunk muscle activity was directly affected by the amount of warning/preview time.

Sudden loading can occur as people handle materials within which loads can shift. As the center of mass shifts during lifting, the lifter's body tries to compensate for the load shifting perturbation, which in turn affects the biomechanical loads experienced during the lifting task. This may partially explain why workers who handle beverage containers were found to have a higher incidence rate of low back injury compared to other jobs that involves frequent lifting (McGlothlin, 1996). In a laboratory-based biomechanical study, Pinto (2013) showed that, relative to a stable load, when subjects were asked to transfer a container of water at hip level from left to right, the liquid load significantly increased muscle activity in the erector spinae and external oblique muscles as the load crossed the middle of the body and was moved to the target destination. 
Others have shown that a lifter's muscle activity and range of motion could be affected by the shifting of a solid mass within the lifted container. For example, Lee et al. (2002a) carried out a study by having an iron cylinder in a wooden box which could generate a sudden load when the cylinder shifted in posterior-anterior direction inside the box. These authors found that load shifting increased the total lifting time, linear length of the center of pressure, as well as the relevant muscular contraction levels (Lee, 2002a). Also, the impact caused by load shifting could trigger larger peak exertions during the lifting task as evidenced by Lee's (2002b) finding that the maximal exertions for trunk muscles, including the erector spinae, occurred about $0.2 \mathrm{~s}$ after the load shifted.

While Lee's studies (2002 a and b) looked at sagittally symmetric lifts, and Pinto's (2013) study looked at horizontal transfers at hip level, there do not appear to be any prior studies investigating unstable lifting conditions that included both a vertical lifting component and an asymmetric placement component. Thus, the study presented in this paper used a lifting task that included both vertical and horizontal movements of the lifted box. Moreover, given the effects of visual information on the sudden loading response, this study investigated the contribution of visual access to the contents of the box. In other words, does the muscle response change when lifting an unstable load and when the material handler does not have visual access to the contents of the box. The general hypothesis was that lifting a covered unstable load would increase the biomechanical loads experienced by the lifter. Specifically it was hypothesized that, relative to lifting a stable load, increased muscle activities and biomechanical responses would be found (1) when lifting containers of liquid in a box, (2) when lifting a solid 
mass that could, but does not, shift position during the lift, and (3) when lifting a box where the solid mass does shift position during a lift. It was further hypothesized that having visual access, for example, lifting an uncovered box, where the person lifting could see the contents of the box, would mitigate these effects. 


\section{Methods \\ Participants}

Fourteen subjects, eight male and six female (Height: $178 \pm 3.1 \mathrm{~cm}$, Weight: $168 \pm 8.8$ lbs. Age: $21.8 \pm 0.9$ ) participated in the study. Exclusion criteria included significant back, leg, neck or shoulder pain in the past 12 months, back surgery or limiting clinical conditions, or pregnancy. None of the participants did intensive physical activity 24 hours before participating in the study and none of the participants was currently working in any occupation that requires heavy material handling. All participants signed a consent form that had been approved by the University's office of responsible research practices.

\section{Experimental Design}

In this study, participants lifted a box from a position directly in front of the body to a location at approximately elbow height on the participant's left side (90 degrees of asymmetry). There were two primary independent variables in this study: (1) the stability of the load in the box and (2) visual information the lifter had about the stability of the load. The three levels of load stability were manipulated by (1) placing a stable load in the box, (2) placing containers of liquid that could shift as the box was lifted, (3) placing an unstable solid mass (a bowling ball) positioned in the center of the box in a small holder, depending upon how the box was moved, the load may or may not have 
shifted during box movement. This latter condition was then subdivided in the analysis into two conditions: one where the ball moved and one where the ball did not move. The visual access was controlled by having a cover over the wooden box that prevented the participant from seeing the contents of the box.

The dependent measures were comprised of spine kinematic and kinetic measures (the moments at the base of the spine), electromyographic measures, lifting duration, and the frequency of load shifting occurrences (when handling the boxes with the bowling ball). Specifically, the kinematic measures provided the spine movements, $\mathrm{T} 1$ relative to L5/S1, in the three cardinal planes: (1) forward flexion, (2) lateral bending and (3) twisting. Likewise, the three dimensional moments acting on the spine at $\mathrm{L} 5 / \mathrm{S} 1$ were obtained relative to these three planes of motion. Electromyographic (EMG) signals obtained from surface electrodes were collected from the left and right (1) Anterior deltoid (ADL, ADR), (2) Biceps (BCL, BCR), (3) Erector spinae (ERSL, ERSR), and (4) External oblique (EXOL, EXOR) muscles. For each lifting task, the lift duration and, if the lifting task was a condition that included the ball, whether or not the ball shifted during the lift were recorded.

\section{Apparatus}

Spinal movements and moments, in all three dimensions, were collected using a magnetic motion capture system (Motion Monitor by Innsport, Chicago, IL, USA). The participant stood on two force platforms that measured the ground reaction forces during the lifting tasks. Electromyographic (EMG) data were obtained using a wireless surface EMG system (Trigno by Delsys) with sampling rate of $2000 \mathrm{~Hz}$. The wooden box lifted 
by the participant weighed $4.54 \mathrm{~kg}$. The contents of the box weighed an additional $6.8 \mathrm{~kg}$ and, depending upon the experimental condition, were comprised of books, bags of water, or a bowling ball.

An accelerometer on the box was used to capture when the lift was initiated and whether the ball shifted inside of the box during the lifting task. A force scale was used to capture when the lifting task was completed.

\section{Procedures}

After signing the informed consent document, electrodes were placed over the muscle of interest as follows: (1) Erector Spinae at the L3 level approximately $3 \mathrm{~cm}$ lateral to the spinous process, (2) Biceps midway between the shoulder and the elbow over the belly of the muscle, (3) Anterior Deltoid over the belly of the muscle, and (4) External Oblique approximately $15 \mathrm{~cm}$ lateral to the umbilicus and at an angle about 45 degrees relative to the vertical (Konard, 2005). Participants performed a series of isometric maximal contractions using a strength-testing apparatus to normalize the EMG activity collected during the actual lifting trials. The techniques used for MVC testing simulated the body position where the maximal muscle activities would be expected in later lifting task. Although there is possibility the muscle activation in those exertions could be less than the actual maximal capacity, the data analysis after is with-in subject comparison, thus it was decided to use those values as reference.

Using tape and straps, motion capture sensors were attached bilaterally on the shanks, thighs and over the superior end of the sacrum and the first thoracic vertebra. An upright "neutral" posture was sampled for each participant as a reference posture. 
Participants were instructed to lift a wooden box from a location on the floor at $20 \mathrm{~cm}$ in front of the participant's toes to a surface positioned $75 \mathrm{~cm}$ above the floor at the participant's left side (Figure 1). The distance to the edge of the destination shelf was about $30 \mathrm{~cm}$ from the lateral side of subject's left foot. The participants were instructed not to move their feet when lifting.

The participants lifted the stable load 5 times, the unstable liquid load 5 times and unstable solid mass 10 times when the box was uncovered and the same number of lifts when the box was covered. The unstable solid mass load was placed in a shallow frame at the center of the wooden box. Depending on how the box was lifted, this may have resulted in the solid mass shifting during the lift. In total, each participant lifted the box 40 times to complete all experimental conditions. Lifts were performed at a rate of approximately one per minute. The sequence of blocks of load stability conditions (stable, liquid, bowling ball) and load visibility conditions were randomized across participants. 


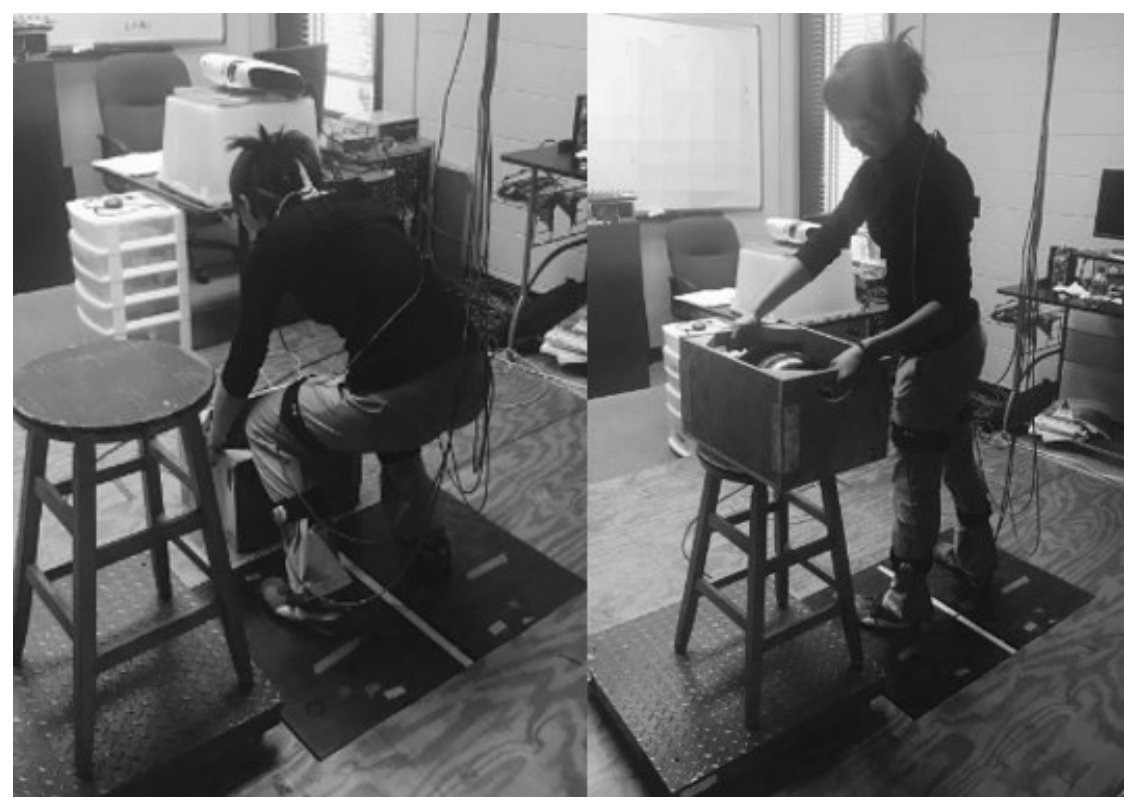

Figure 1. Experiment Set Up

\section{Data analysis}

Digital smoothing algorithms was applied to the raw EMG which included obtaining a root mean square (RMS) transformation for each muscle's data. The normalized 90-percentile value for each muscle sampled during each lifting task was obtained using Matlab code, aiming at capturing the peak muscle activities during the work (Jonsson, 1982). Across the trials for each experimental condition, both the average and maximum value of these 90th percentile values were calculated. Statistical analyses were based on the compiled maximal 90th percentile values (Max90) and average 90th percentile values (Avg90). For spine kinematic and kinetic measures, the peak values from each lifting task were extracted. The data analyses used the peak of the peak (PoP) values and the average of the peak (AvgP) values. 
A series of pair-wise two-way ANOVA's were used to assess the effect of different load stability conditions (stable, liquid, non-rolling ball, and rolling ball) conditions and the visual access conditions. All these models were within-subject analyses (Table 1). The effects of visual access on the ball rolling frequency was assessed using applied Chi-square analysis.

\begin{tabular}{|l|l|l|l|l|l|l|l|l|l|l|l|}
\hline & Uncovered & Covered & & Uncovered & Covered & & Uncovered & Covered & & Uncovered & Covered \\
\hline Stable & & & Stable & & & Stable & & & $\begin{array}{l}\text { Non- } \\
\text { rolling }\end{array}$ & & \\
\hline Liquid & & & $\begin{array}{l}\text { Non- } \\
\text { rolling }\end{array}$ & & & Rolling & & & Rolling & & \\
\hline
\end{tabular}

Table 1. Pair-wised Comparison between Different Load Stability and Visual Access Conditions 


\section{Results}

\section{Comparison between stable and liquid load}

The comparison between the stable vs liquid conditions did not indicate significant changes in the EMG, posture, or moment measures. Moreover, there were no differences in the lifting duration due to either the visual access or the load stability.

Looking across both the stable load and the liquid load conditions, visual access significantly reduced $(\mathrm{p}<0.05)$ the Max90 muscle activities in the left anterior deltoid (Figure 1) visual access. Similar trends were found for the right anterior deltoid $(\mathrm{p}<0.1)$ and right erector spinae $(\mathrm{p}<0.1)$ muscles.

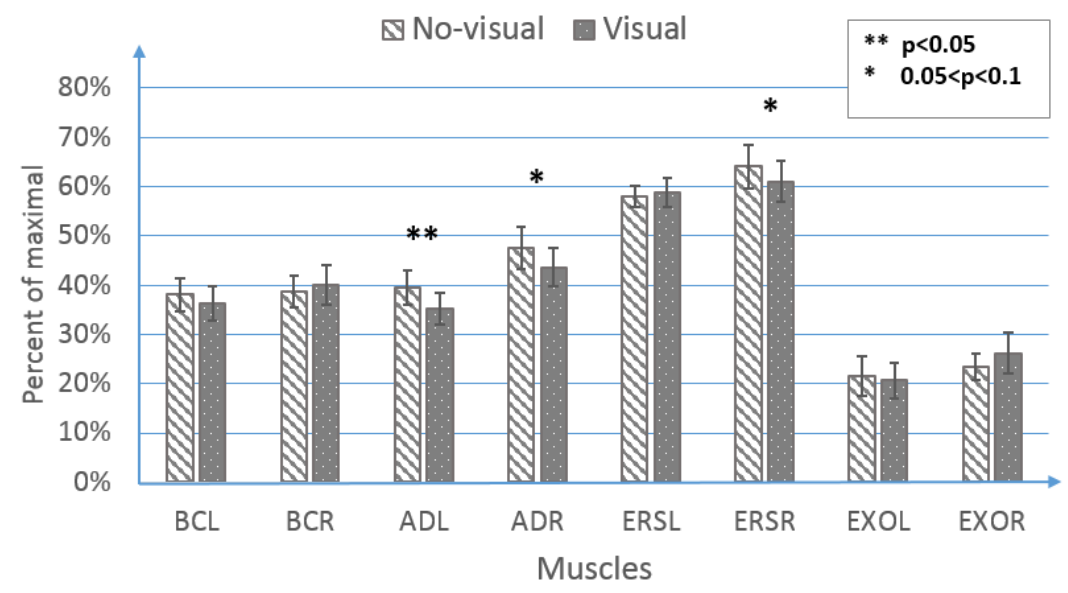

Figure 2. Visual Access Effect on Max 90 Muscle Activities for Data Collected During the Stable and Liquid Handling Trials 
As for changes in the movement kinematics, the analysis of both the maximum (PoP) and average values (AvgP) showed that visual access increased the thoracic rotation and lateral flexion. The thoracic lateral flexion moment was also higher in lifting tasks when subjects had visual access to the load (Figure 3a-d).

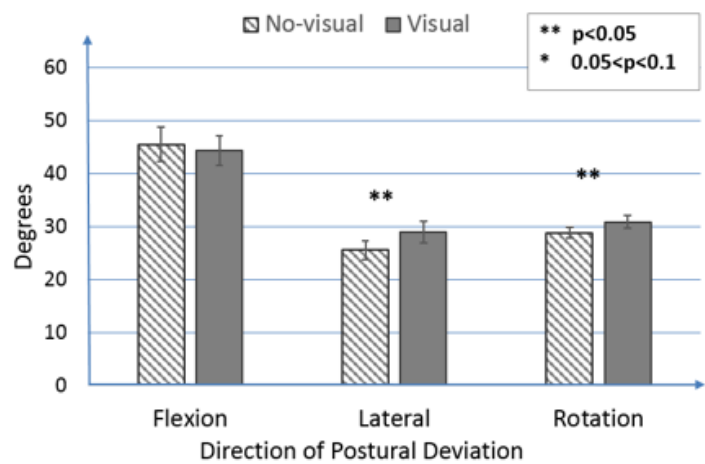

(a)

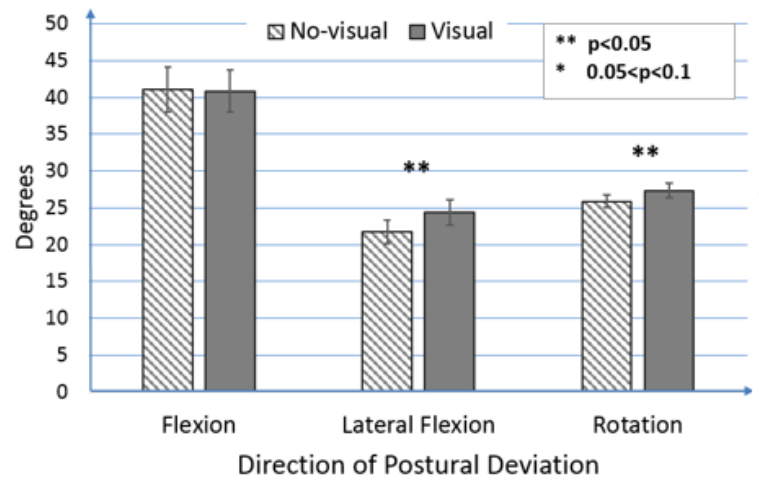

(c)

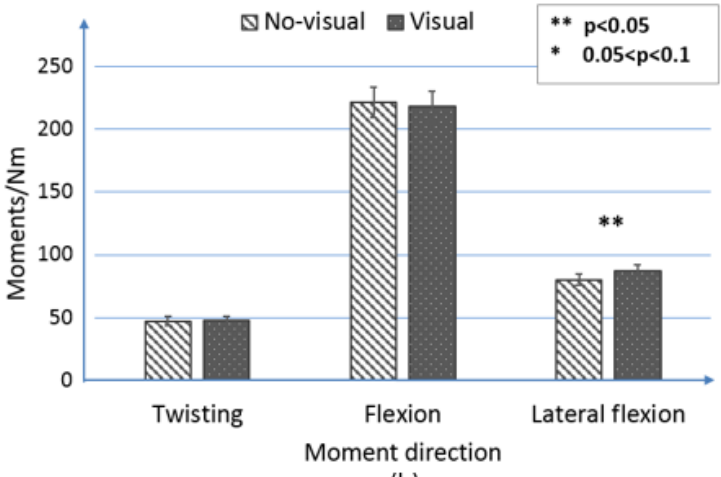

(b)

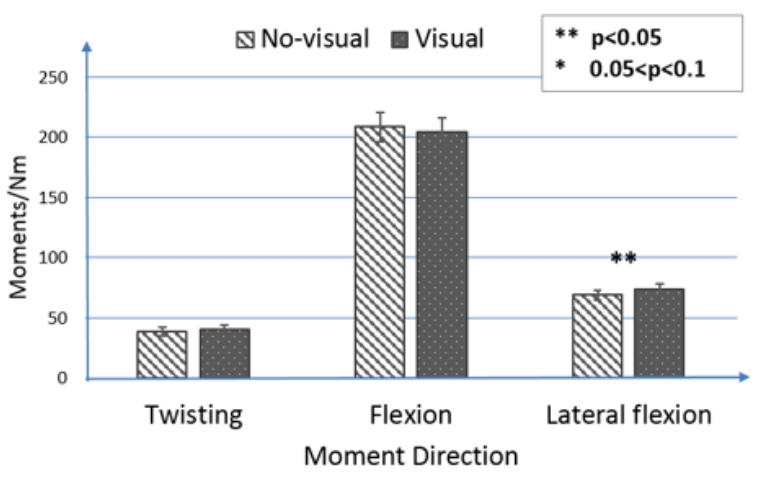

(d)

Figure 3. Visual Access Effect on Thoracic Biomechanics Responses - Stable vs Liquid for Pop $(\mathrm{a}, \mathrm{b})$ and $\operatorname{AvgP}(\mathrm{c}, \mathrm{d})$ Analyses. 
Comparison between stable and potentially unstable load (Lifts where the ball did not roll)

The potentially unstable load (i.e. non-rolling condition) had a significant effect on lifting duration in which the non-rolling trials took 6.5 percent longer to complete than lifts that were known to be stable $(\mathrm{p}<0.05)$. Further analysis showed that the time difference was due to the horizontal velocity of the box during the second stage of the lifting task, which was defined as the box movement in the transverse plane. On average, subjects moved the box horizontally 11 percent faster when lifting the stable load compared to the potentially unstable load $(\mathrm{p}<0.05)$. The load stability also affected the Avg90 and Max90 muscle activity levels in the left biceps and right anterior deltoid. Both muscles had a reduced response to the conditions when the load was potentially unstable (Figure 4). No differences between these stability conditions were found in either the kinematic or the moment data. 


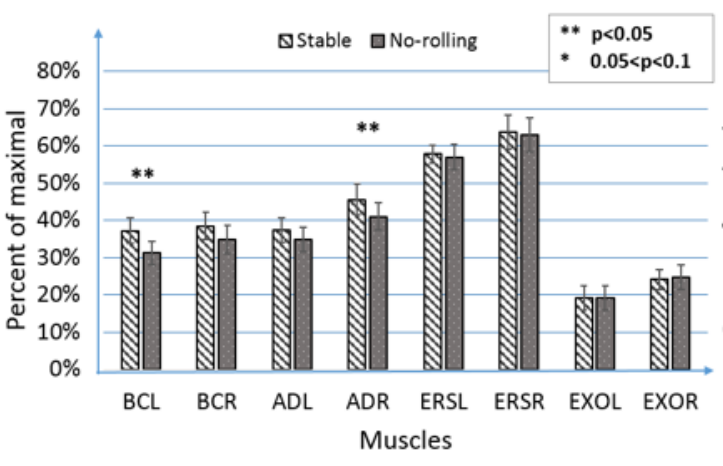

(a)

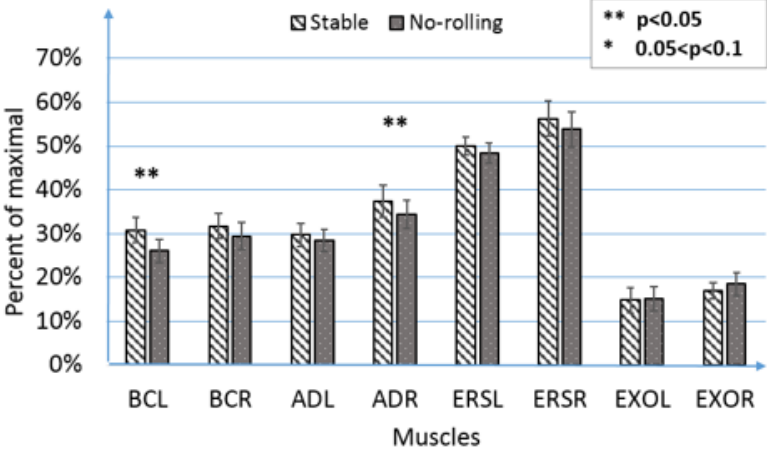

(b)

Figure 4. The Load Stability Effect on Muscle Activities - Stable vs. Non-rolling for Max90 (a) and Avg90 (b) Analyses.

Visual access significantly reduced muscle activity in the right anterior deltoid in the analysis of the Max90 values. When looking at the averaged 90th percentile values (Avg90), visual access significantly reduced the peak responses in the left erector spinae muscles and marginally reduced the peak responses in the right erector spinae muscles. (Figure 5). The only kinematic change with visual access was an increase in the most extreme lateral spine flexion values, which increased approximately 3 degrees (from 24.8 degrees without visual access to 27.9 degrees $)$ with visual access $(\mathrm{p}<0.05)$. 


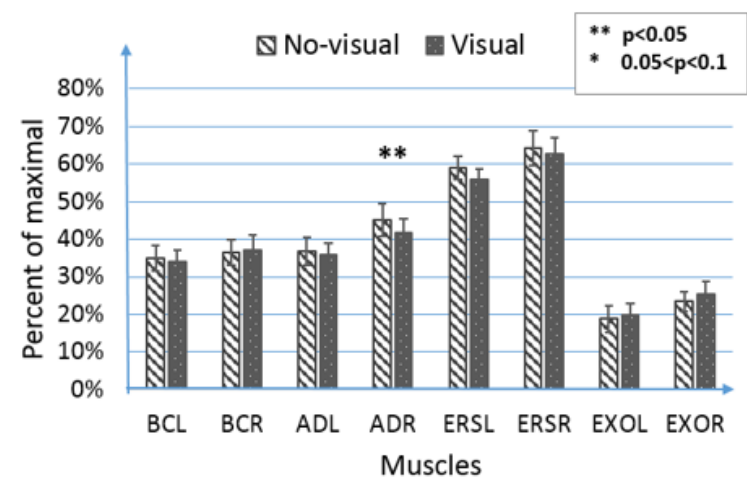

(a)

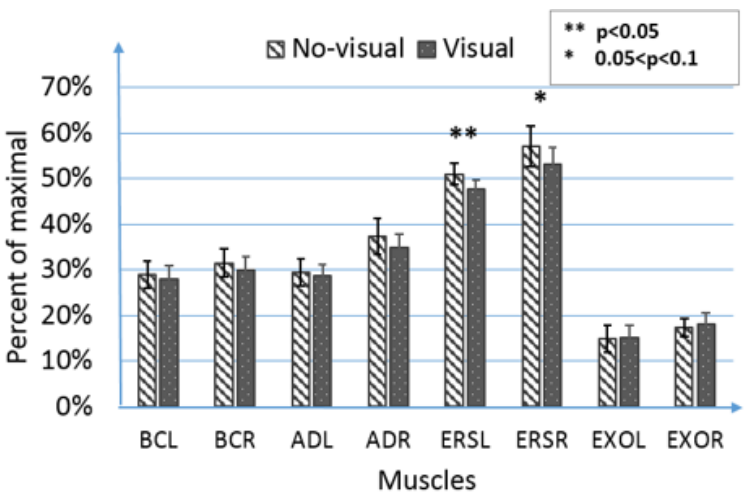

(b)

Figure 5. Visual Access Effect on Muscle Activities - Stable vs. Non-rolling for Max90 (a) and Avg90 (b) analyses.

\section{Comparison between stable and unstable load (rolling ball)}

The unstable load significantly increased thoracic lateral flexion by 2.8 degrees (p-value $<0.05$ ) in the AvgP analysis. It also significantly increased the lifting duration by $6.6 \%$ (p-value $<0.05)$. However, no load stability effect on muscle activity was found in stable vs rolling condition comparison for the Avg90 or the Max90 data sets.

Visual access had similar effects on the Avg90 and Max90 analyses when comparing muscle activation levels in stable vs unstable load lifting tasks. The covered box significantly increased the muscle activities in the left biceps, both anterior deltoid and both erector spinae muscles (Figure 6). When analyzing the result for rolling cases only, this analysis of the rolling cases also showed that visual access (i.e. the box was not covered) reduced the responses in all the muscles on the left side $(p<0.05)$. 


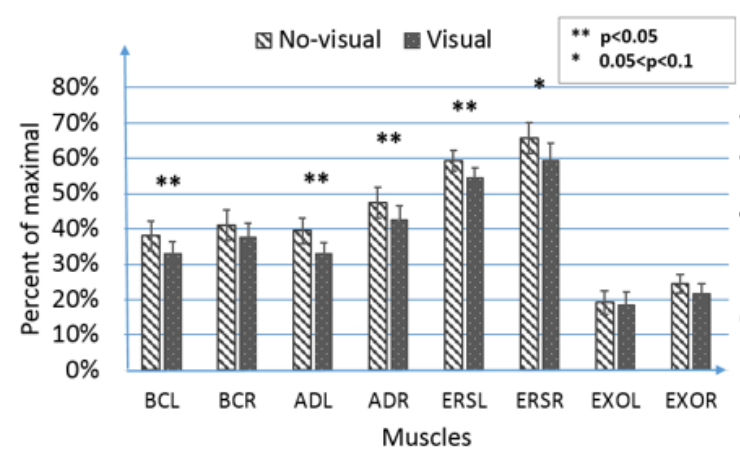

(a)

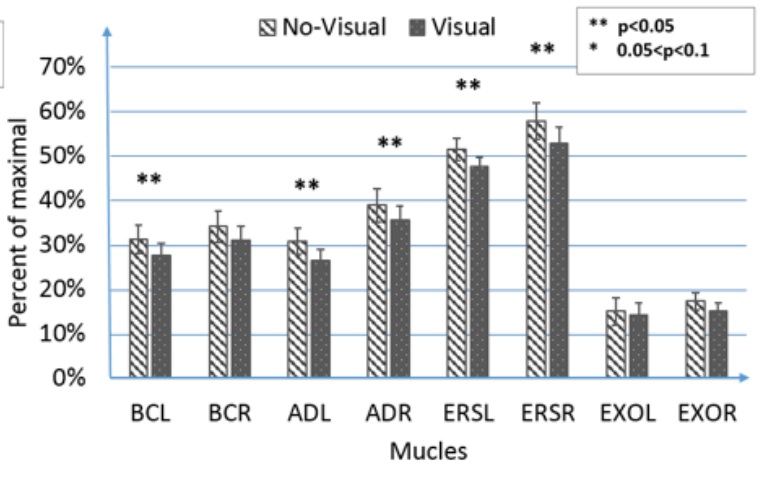

(b)

Figure 6. Main Effect for Visual Access on Muscle Activities for the- Max90 (a) and Avg90 (b) Analyses (Averaged Across the Stable and Rolling Conditions)

Comparison between potentially unstable (non-rolling) and unstable (rolling) load conditions

When analyzing the trials in which the bowling ball was lifted, ball rolling led to higher Avg90 muscle activities in the right biceps $(\mathrm{p}<0.05)$ and right anterior deltoid $(\mathrm{p}<0.01)$. At the same time, left external oblique had significantly lower muscle activity in the lifts where the ball rolled. The right external oblique muscle showed a similar trend. No effect of visual access or load stability was found on the biomechanical responses or the lifting duration. 


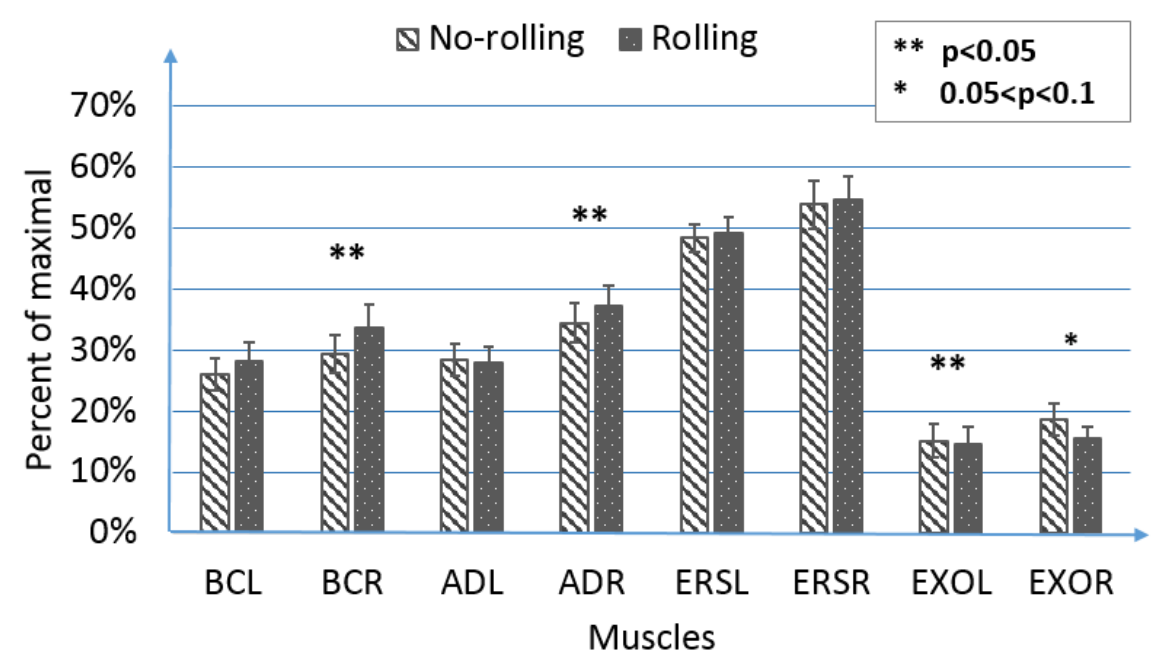

Figure 7. Load Stability Effect on Muscle Activity - Non-rolling vs. Rolling (Avg90)

When lifting the box with the bowling ball, the frequency of the ball shifting inside the box was significantly higher when the box was covered $(\mathrm{p}<0.05)$. However, irrespective of whether the ball moved, figure 7 shows that the Avg90 muscle activities were significantly reduced when the box was uncovered for the left bicep $(\mathrm{p}<0.01)$, left $(p<0.05)$ and right $(p<0.01)$ anterior deltoid, left erector spinae $(p<0.01)$ and left external oblique $(\mathrm{p}<0.05)$. There was also a similar trend in the right erector spinae data $(\mathrm{p}<0.1)$. 


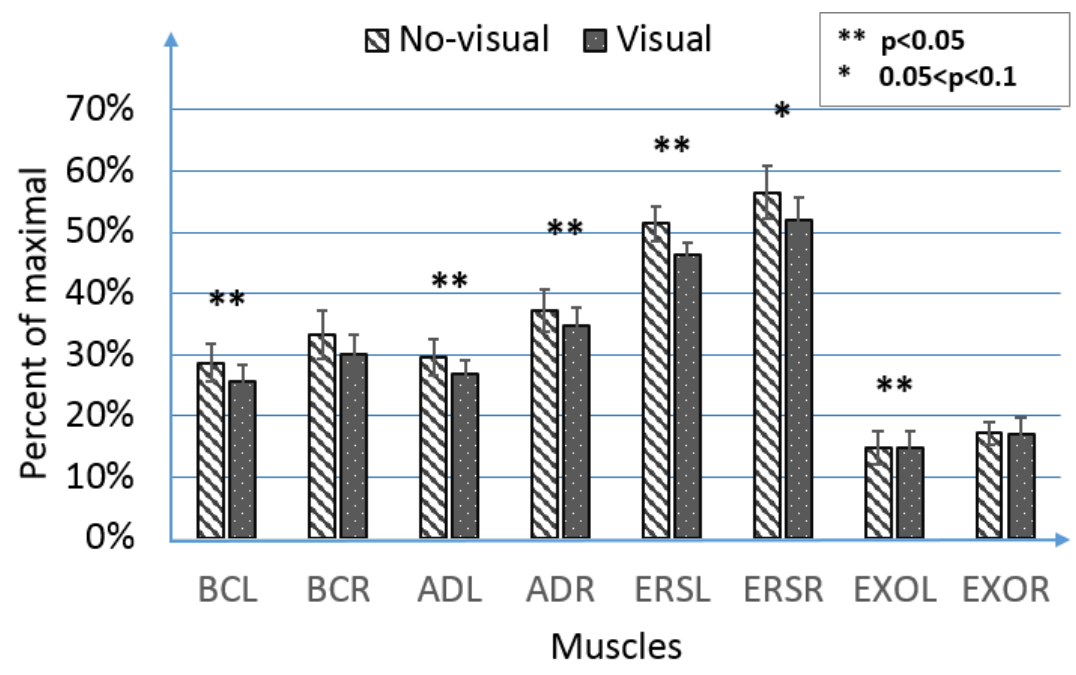

Figure 8. Visual Access Effect on Muscle Activities - Non-rolling vs Rolling (Avg 90)

\section{Interaction Effects}

In this study, there was very limited interaction between visual access and load stability. Only one significant interaction effect was found and this was for the right erector spinae when comparing the lifts of stable and liquid loads. Muscle activity in the right erector spinae showed no difference between the stable and liquid loads when subjects had visual access to the load, however, the right erector spinae showed more activity in the stable condition when the box was covered and the subjects had no visual access. 


\section{Discussion}

The general hypothesis that lifting an unstable load without visual access during the lifting task would increase the biomechanical loads experienced by the lifter was only partially supported. However, the results indicate that an interesting relationship exists between visual access and the potential for load instability.

\section{Comparison between stable and liquid}

Contrary to our hypothesis, the current study did not show a significant difference in muscle activity or other biomechanical measures when lifting stable versus liquid loads. This is in contrast to a similar study that found that a liquid load significantly increased muscle activity in the erector spinae and external oblique muscles during the second phase of their lifting tasks (Pinto, 2013). Potential reasons for the difference could be: 1) the container used in the current study was of limited size $(40 \times 25 \times 30 \mathrm{~cm})$, and was not directly filled with liquid. Instead, the liquid was kept in two bags, which were then placed in the container. This could reduce the potential for the water to shift during the lifting task; 2) the lifting task in this study required the container to be moved vertically from floor level to hip level, whereas Pinto's study required only horizontal movement of the container. There was less potential for the water to shift during the vertical component of the lifting task. 
It was found that visual access increased the twisting and lateral bending of the spine and also increased lateral flexion moment acting on the spine. When the two bags of water were visible, the participants seemed to adopt some behaviors that afforded better control the load and reduce the potential of load shifting.

Given the limited ability of the liquid to shift within the container it is not surprising that no significant differences were found in the muscle activities when comparing the stable and liquid lifting tasks. However, the interaction effect, where the covered box led to significantly higher right erector spinae activity when the stable load was lifted could reflect the uncertainty that the participants had when lifting the liquid. Further study is needed to understand the basis for this interaction effect.

\section{Comparison between stable and non-rolling}

Compared to the stable and rolling load conditions, the non-rolling conditions could be considered a special situation in which the potentially unstable load remained "stable" by the subjects' active effort to control the load's stability. Thus, it was defined as a separate load stability condition in this study where it was found that lifting these potentially unstable loads led to higher muscle activities in the left biceps and the right anterior deltoid muscle. Given that the experimental task required subjects to lift the box in front and place it at hip level on their left side, increased activation of these muscles during this asymmetric lift could help keep the box level during the asymmetric lifting task. It was surprising that the trunk muscles were not found to differ in their response across these experimental conditions, as we had hypothesized there would be increased cocontraction of the trunk musculature to further maintain a smooth and stable movement 
of the box. However, it was found that the duration of the lifting task, or more specifically the duration of horizontal movement of the box for unstable load lifting, was longer compared with the duration during the stable load lifting task. This difference in lifting time could potentially explain the absence of a load stability effect on relevant measurements in this study. If we considered visual access as a type of warning signal, Mawston's (2007) study, which assessed the effects of a warning signal prior to a sudden loading event occurring during a lifting task, found that a warning signal did not alter the level of trunk muscle activity prior to the sudden loading, although it did reduce the response latency. Lavender et al. (1989) reported decreased peak activities in erector spinae muscles during a sudden loading even as the amount of preview time prior to a sudden loading event increased from 100 to $400 \mathrm{~ms}$. It should be noted that in both of these sudden loading studies participants would likely have some expectation of the load change regardless of the presence of a warning. In contrast, in the current study the sudden shift in the load due to the ball rolling within the box did not always occur. Even so, we found a similar beneficial effect of visual access on muscle activities when handling the potentially unstable load.

\section{Comparison between stable and rolling}

The comparison between the stable load condition and the condition where the ball rolled showed no differences in the muscle activities. In a former study, it was also found that unstable loads had no effect on the agonist muscle activity when the subjects lifted at their preferred pace (Pinto, 2011). However, in Pinto's study (2011), unstable loads did increase the muscle activity in the erector spinae and external oblique muscles 
when subjects were required to lift at a fast pace. In the current study, the lifting speed was not controlled, therefore we could assume participants lifted at their preferred pace. The unstable loads did result in slower lifts as the total lifting time was increased, which could have masked the effect of load stability on muscle responses. A cursory analysis on the vertical acceleration of the box at the beginning of the lift did not reveal any differences in the speed of box movement and, therefore, suggests the additional time when lifting unstable loads in this study was associated with the lateral motion of the box and its placement on the stool.

As described in the results section, visual access significantly reduced the activity in several muscles (left biceps, anterior deltoid ( $\mathrm{E} \& \mathrm{R}$ ) and erector spinae (L \& R)) across all lifts in which the box contained the bowling ball. Similar results were found by Looze (2010) who had people lifting boxes weighing $6.5 \mathrm{~kg}$ or $16.5 \mathrm{~kg}$, while in certain conditions the subjects could only anticipate the range of load weight. In Looze's study, it was found that 'unknown' load weight significantly increased back muscle activation and peak L5/S1 torque. Similarly, in Heiss's study (2001), subjects were asked to lift similar weights that immediately followed with a lighter or heavier load. It was found that overestimation of the load weight could increase peak lumbar extensor moments. The result from previous studies suggested the necessity of knowledge about load weight as well as the potential for load weight change. In the current study, especially for the rolling conditions, lower muscle activity in those trials with visual access suggests that when lifting unstable loads, having visual access, for example by a translucent container lid that provides visual access for the lifter, could possibly help reduce the muscle 
response, the development of fatigue if this is part of a repetitive task, and the potential for muscle injury.

\section{Comparison between non-rolling vs rolling}

When lifting an unstable load (in the current study a bowling ball in a box), whether or not it rolled, visual access reduced the activity in several muscles (left bicep, anterior deltoid (L\&R), erector spinae (L\&R), and left external oblique). These findings are consistent with prior studies showing reduced muscle activity when a sudden load could be seen before it was experienced (Marras et al., 1987; Lavender et al., 1989).

At the point of the lift initiation, the loads had the same degree of instability, subjects lifted at the same speed and there was no lift duration difference between the rolling and non-rolling conditions. When the load did shift, it significantly increased the activation of the right biceps and the right anterior deltoid muscles while at the same time decreased the activation of the left (and right) external oblique muscles. This finding is consistent with a former study, that found that lifting an unstable load significantly increased the muscle contraction level in biceps and erector spinae (Lee \& Lee, 2002). It suggests that when handling an unstable load, the load imbalance generated by the load shifting could increase the exertion in agonist muscle groups. As for the reduced external oblique activity in the ball rolling condition, it could be that in the non-rolling trials the subjects recruited the external oblique muscles to a higher level to enhance the stability, thus generating a "non-rolling" result.

There are several limitations in this study. 1) There was no real-time capture of the directionality of the load shifting. This type of data could have explained some of the 
variation in the muscle response data. 2) The degree of instability of the liquid load was limited due to the container design and the use of bags to contain the liquid. More open containers, where the liquid could shift more, would be expected to show more of a load stability effect. 3) No experimental control of the lifting pace was used. While this may have masked some effects as subjects' adopted a slower lifting pace when handling unstable objects, lifting behaviors that are not time paced may have more external validity. 


\section{Conclusion}

The hypothesis that lifting unstable loads would increase activities in certain muscles such as biceps and anterior deltoid, during a lifting task was not supported.

However, this study did find that when handling an unstable load, being able to see the load reduced the load shifting possibility and the magnitude of the muscular responses. This indicated that in industry, it might be beneficial to use containers with transparent covers where loads could possibly shift inside. These covers would allow workers to see the contents inside which could reduce the physical demands and the potential for musculoskeletal disorders. 


\section{Reference}

[1] Andersson, G.B.J. The epidemiology of spinal disorders. In J.W. Frymoyer (ed): The Adult Spine. Lippincott-Raven, 1997, 93-141.

[2] Anderson, G. B., 1981, Epidemiologic aspects of low back pain in industry. Spine, 6, 53-60.

[3] Bureau of Labor Statistics 2016: https://www.bls.gov/iif/oshwc/osh/case/ostb4754.pdf [4] Heiss DG, et al. "Anticipatory control of vertical lifting force and momentum during the squat lift with expected and unexpected loads." J Orthop Sports Phys Ther, 2001, 31(12):708-23.

[5] Jonsson B. Measurement and evaluation of local muscular strain in the shoulder during constrained work[J]. Journal of human ergology, 1982, 11(1): 73-88.

[6] Konrad P. The abc of emg[J]. A practical introduction to kinesiological electromyography, 2005, 1: 30-35.

[7] Lavender S A, Mirka G A, Schoenmarklin R W, et al. The effects of preview and task symmetry on trunk muscle response to sudden loading [J]. Human Factors: The Journal of the Human Factors and Ergonomics Society, 1989, 31(1): 101-115.

[8] Lee Y H, Lee T H. Human muscular and postural responses in unstable load lifting [J]. Spine, 2002, 27(17): 1881-1886. 
[9] Lee Y H, Lee T H. Muscle response while holding an unstable load [J]. Clinical biomechanics, 2002, 17(4): 250-256.

[10] De Looze, M. P., et al. "Trunk muscle activation and low back loading in lifting in the absence of load knowledge." Ergonomics 43.3 (2000): 333-344.

[11] Marras W S, Rangarajulu, S L, Lavender S A. Trunk loading and expectation [J]. Ergonomics, 1987, 30(3): 551-562.

[12] Mawston G A, McNair P J, Boocock M G. The effects of prior warning and liftinginduced fatigue on trunk muscle and postural responses to sudden loading during manual handling[J]. Ergonomics, 2007, 50(12): 2157-2170.

[13] McGlothlin, J.D., 1996. Ergonomic Intervention for the Soft Drink Beverage Delivery Industry. U.S. Department of Health and Human Services (DHHS), Centers for Disease Control and Prevention (CDC), The National Institute for Occupational Safety and Health (NIOSH). No. 96e109.

[14] Molumphy M, Unger B, Jensen G M, et al. Incidence of work-related low back pain in physical therapists[J]. Physical therapy, 1985, 65(4): 482-486.

[15] Owen B D, Frazier Damron C. Personal characteristics and back injury among hospital nursing personnel[J]. Research in nursing \& health, 1984, 7(4): 305-313. [16] Pinto V. The Effect of Unstable Loads, Lifting Pace, and External Weight on Trunk Muscle Activity during Asymmetric Lifting in the Transverse Direction [D]. NEW YORK UNIVERSITY, 2011. 
[17] Pinto V J, Sheikhzadeh A, Halpern M, et al. Assessment of engineering controls designed for handling unstable loads: An electromyography assessment[J]. International Journal of Industrial Ergonomics, 2013, 43(2): 181-186. 